\title{
Protocolo de revisão sistemática da literatura: dados em saúde oncológica
}

\author{
Systematic literature review protocol: data on cancer health \\ Protocolo de revisión sistemática de la literatura: datos sobre la salud del cáncer
}

Recebido: 14/10/2021 | Revisado: 23/10/2021 | Aceito: 01/11/2021 | Publicado: 02/11/2021

Nathália Varano
ORCID: https://orcid.org/0000-0002-7761-9181
Universidade Federal de Uberlândia, Brasil
E-mail: nathalia.varano@ufu.br
Mariane Bruna da Silva Mendes
ORCID: https://orcid.org/0000-0002-7529-3720
Universidade Federal de Uberlândia, Brasil
E-mail: mariane.bruna@ufu.br
Bárbara Mendes de Oliveira
ORCID: https://orcid.org/0000-0001-7258-3389
Universidade Federal de Uberlândia, Brasil
E-mail: babimendes203@gmail.com
Tatiana Gonçalves dos Reis
ORCID: https://orcid.org/0000-0002-1172-2987
Faculdade Uberlandense de Núcleos Integrados de Ensino, Serviço Sociale Aprendizagem, Brasil
E-mail: tatigr@gmail.com
Cristiane Martins Cunha
ORCID: https://orcid.org/0000-0002-6748-8587
Universidade Federal de Uberlândia, Brasil
E-mail:cristiane.cunha.ufu@gmail.com
Bruna Helena Mellado
ORCID: https://orcid.org/0000-0002-5207-7689
Universidade Federal de Uberlândia, Brasil
E-mail: bru.mell@usp.br

\begin{abstract}
Resumo
Justificativa e objetivo: A revisão sistemática de estudos que versam sobre o autocuidado de mulheres submetidas a mastectomia é crucial para avaliar a segurança e a efetividade das intervenções assistenciais prestadas a esta parcela da população. O objetivo deste artigo é apresentar um tutorial para o planejamento e execução de revisões sistemáticas de forma coesa, seguindo um fluxo de trabalho dinâmico e em equipe. Métodos: A revisão sistemática da literatura é o tipo de pesquisa que organiza, crítica e integra as evidências disponíveis na literatura. A informação divulgada nos artigos nem sempre está livre de vieses, e para minimizar essas interferências é necessário que uma revisão de literatura contemple itens como o delineamento, o registro do protocolo, a execução, a análise dos resultados e a ampla divulgação. Para manter a coesão acerca das revisões sistemáticas pesquisadores têm usado cada vez mais o fluxograma PRISMA, que têm melhorado a qualidade dos relatos das revisões sistemáticas, pois fornece uma lista de itens a serem descritos, e este artigo enfatiza os principais passos para a execução de uma revisão sistemática. Considerações finais: As evidências científicas obtidas através de uma revisão sistemática de literatura, bem desenhada, pode proporcionar ao profissional da saúde maior confiança no momento da prática clínica, aprimorar os benefícios aos pacientes assistidos e servir como ferramenta para auxiliar os gestores, ainda pode corroborar à implantação de novas estratégias em prol da saúde geral.
\end{abstract}

Palavras-chave: Revisão sistemática; Câncer de mama; Oncologia; Enfermagem.

\begin{abstract}
Background and objective: The systematic review of studies that deal with the self-care of women undergoing mastectomy is crucial to assess the safety and effectiveness of care interventions provided to this portion of the population. The purpose of this article is to present a tutorial for planning and executing systematic reviews in a cohesive way, following a dynamic, team-based workflow. Methods: A systematic literature review is the type of research that organizes, critiques and integrates the evidence available in the literature. The information disclosed in articles is not always free from bias, and to minimize these interferences it is necessary that a literature review includes items such as design, protocol registration, execution, analysis of results and wide dissemination. To maintain cohesion about systematic reviews, researchers have increasingly used the PRISMA flowchart, which has improved the quality of reports from systematic reviews, as it provides a list of items to be described, and this article emphasizes the main steps for carrying out a systematic review. Final considerations: The scientific evidence obtained through a well-designed systematic literature review can provide the health professional with greater confidence at the time of clinical practice,
\end{abstract}


improve the benefits to assisted patients and serve as a tool to assist managers, it can also corroborate to the implementation of new strategies in favor of general health.

Keywords: Systematic review; Breast cancer; Oncology; Nursing.

\begin{abstract}
Resumen
Antecedentes y objetivo: La revisión sistemática de los estudios que abordan el autocuidado de las mujeres sometidas a mastectomía es fundamental para evaluar la seguridad y eficacia de las intervenciones de atención que se brindan a esta porción de la población. El propósito de este artículo es presentar un tutorial para planificar y ejecutar revisiones sistemáticas de manera coherente, siguiendo un flujo de trabajo dinámico y en equipo. Métodos: Una revisión sistemática de la literatura es el tipo de investigación que organiza, critica e integra la evidencia disponible en la literatura. La información divulgada en los artículos no siempre está libre de sesgos, y para minimizar estas interferencias es necesario que la revisión de la literatura incluya ítems como diseño, registro del protocolo, ejecución, análisis de resultados y amplia difusión. Para mantener la cohesión sobre las revisiones sistemáticas, los investigadores han utilizado cada vez más el diagrama de flujo PRISMA, que ha mejorado la calidad de los informes de las revisiones sistemáticas, ya que proporciona una lista de elementos a describir, y este artículo enfatiza los pasos principales para llevar a cabo una revisión sistemática. Consideraciones finales: La evidencia científica obtenida a través de una revisión sistemática de la literatura bien diseñada puede brindar al profesional de la salud una mayor confianza en el momento de la práctica clínica, mejorar los beneficios para los pacientes asistidos y servir como una herramienta para ayudar a los administradores, también puede corroborar la implementación de nuevas estrategias a favor de la salud general.
\end{abstract}

Palabras clave: Revisión sistemática; Cáncer de mama; Oncología; Enfermería.

\title{
1. Introdução
}

Em uma breve alusão histórica, é possível compreendermos que a atividade de compilar dados científicos na área da saúde acontece há muito tempo, uma das primeiras revisões registradas tem data de 1753, quando Sir James Lind publicou sua pesquisa sobre a prevenção e o tratamento do escorbuto (Library, 2007). No que concerne a primeira soma estatística de estudos científicos, técnica hoje conhecida como metanálise, esta ocorreu em 1904 publicada pelo matemático Karl Pearson (Pearson, 1904).

No entanto, as revisões ditas como sistemáticas começaram a surgir na década de 1950, mas só ganharam consolidação científica no fim da década de 80. Neste espectro, foram marcos a publicação do livro "Efective care during pregnancy and childbirth" e, ainda na década seguinte, houve a criação da Colaboração Cochrane, em Oxford, no Reino Unido (Chalmers \& Enkin \& Keirse 1989); (Cochrane Collaboration, 2012).

Revisar a literatura é uma atividade essencial no tocante ao desenvolvimento acadêmico e científico, cenário onde a revisão sistemática se define como uma síntese rigorosa que busca superar possíveis vieses em todas as etapas de construção de um tema. Cabe salientar que a realização de uma revisão de literatura proporciona evitar a duplicação de pesquisas ou, ainda quando for de interesse, permitir o reaproveitamento e a aplicação de pesquisas em diferentes escalas e contextos (Higgins \& Green, 2011); (Galvão \& Ricarte, 2020).

Vale salientar que as revisões sistemáticas diferem das revisões narrativas ou tradicionais, pois elas apresentam características amplas e carregam informações gerais sobre o tema em questão. Também se distinguem das revisões integrativas, no que concerne aos diferentes delineamentos na mesma investigação, além de expressarem a opinião do(s) próprio(s) autor(s) (Botelho \& Cunha \& Macedo, 2011); (Galvão \& Pereira, 2014).

No tocante a confecção das revisões sistemáticas, estas devem ser abrangentes e não tendenciosas na sua preparação. Os critérios adotados devem ser divulgados para que outros pesquisadores possam ter subsídios para replicar o procedimento metodológico. Revisões sistemáticas de boa qualidade são consideradas o melhor nível de evidência para as tomadas de decisão na prática clínica baseada em evidências científicas (Centre for Reviews and Dissemination, 2009). Pelo fato de seguir um método científico explícito e apresentar resultado novo, a revisão sistemática é classificada como uma contribuição original, na maioria das revistas de pesquisa clínica (Meerpohl \& Herrle \& Antes \& Von, 2012); (Centre for Reviews and Dissemination, 2009); (BAEK et al.,2018). 
Cabe ressaltar que esse recurso metodológico envolve a aplicação de estratégias científicas, com a finalidade de limitar vieses, congregar, avaliar criticamente e sintetizar todos os estudos relevantes que respondem a uma pergunta clínica específica; além disso, promover a atualização dos profissionais de saúde, uma vez que sintetiza amplo corpo de conhecimento e ajuda a explicar as diferenças entre estudos com a mesma questão clínica (Cook, Mulrow \& Haynes, 1997); (Galvão \& Ricarte, 2017).

No que tange à importância da revisão sistemática na enfermagem, estudos apontam que esse recurso pode ser capaz de identificar os efeitos benéficos e nocivos de diferentes intervenções da prática assistencial; além de também poder estabelecer lacunas do conhecimento e identificar as áreas que necessitam de futuras pesquisas, com implicações para a assistência. Ressaltam, ainda, que a revisão sistemática é um recurso valioso de informações para a tomada de decisões (Moloney \& Maggs, 1999).

Outrossim, este protocolo teve como objetivo descrever o passo a passo realizado para a construção de uma Revisão Sistemática de Literatura (RSL) com acadêmicos e docentes de enfermagem de uma Universidade Federal, possibilitando assim um fluxo coeso de confecção deste tipo de pesquisa. Cabe também salientar que este protocolo usou como pano de fundo a RSL realizada em 2020 sobre a compreensão do autocuidado de mulheres com câncer de mama após a mastectomia, dados já publicados.

Destarte, apresentaremos de forma suscinta e descritiva as fases do processo, que são essenciais, para elaborar uma RSL com vistas à saúde oncológica de mulheres com câncer de mama e o trabalho em equipe.

\section{Metodologia}

Foi executada uma pesquisa bibliográfica em que as buscas foram rodadas em três plataformas da web, National Library of Medicine (PubMED), Embase (Elsevier Limited) e Web of Science (Web of Science Group Clarivate), por meio da estratégia desenhada a partir dos seguintes termos indexados em inglês "mulheres com câncer de mama", "autocuidado", "pesquisa qualitativa" e "mastectomia". Os termos indexados utilizados em Ciências da Saúde (DeCS) e suas respectivas traduções, conforme o MeSH terms (Medical Subject Headings) foram: malignant neoplasm of breast; cancer of the breast; breast neoplasms; mastectomy; surgical oncology; surgical procedures, operative; self-management; self-management; management, self; self-care; care, self; self-care; help-seeking behavior; help-seeking behavior; coping behavior; qualitative research; qualitative study; qualitative analysis; thematic analysis; phenomenology; cultural anthropology; hermeneutics; focus group. Para ampliar a abrangência da busca, utilizou-se termos indexados, que foram associados por meio dos operadores booleanos OR e AND.

Em relação a caracterização das pacientes incluídas nos estudos selecionados, tivemos 130 mulheres mastectomizadas, onde a faixa etária predominante oscilou dos 54 a 55 anos, representando 76,15\%. Das 130 mulheres entrevistadas, apenas 114 abordaram seu estado civil Izydorczyk et al.,2019 e Olasehinde et al.,2019, 66,6\% delas eram casas, representando 76 mulheres. Os estudos de Stecher et al.,2019, Dias et al.,2017 e Brendin (1999) não explanaram o estado civil das participantes. Vale ressaltar que todas as mulheres participantes exerciam atividades domésticas e atividades remuneradas.

\section{Equipe de pesquisa}

A seguir, apresentamos a equipe executora da pesquisa, o papel desempenhado por cada pesquisador e suas respectivas afiliações (Quadro 1). 
Quadro 1: Equipe de pesquisa.

\begin{tabular}{|lll|}
\hline Nome do pesquisador & Papel desempenhado & Afiliação \\
Bruna Helena Mellado & Orientador & FAMED-UFU \\
Nathália Varano & Pesquisador & FAMED-UFU \\
Bárbara Mendes de Oliveira & Pesquisador & FAMED-UFU \\
Mariane Bruna da Silva Mendes & Pesquisador & FAMED-UFU \\
Tatiana Gonçalves dos Reis & Pesquisador & FAESSA \\
Cristiane Martins Cunha & Pesquisador & FAMED-UFU \\
\hline
\end{tabular}

Fonte: Autores (2021).

\section{Resultados e Discussão}

\section{Escopo da pesquisa}

A definição sobre as plataformas se deu através de alinhamento entre as bibliotecas virtuais disponíveis na web combinadas àquelas com acesso livre à comunidade acadêmica da Universidade Federal de Uberlândia (UFU).

\section{Critérios adotados para seleção das fontes de busca}

Os critérios adotados para possível seleção dos artigos se deu através de buscas em base de dados de livre acesso, a saber: PubMED, Web of Science e Embase. Também foram realizadas consultas sobre citações e rastreamentos de autores, bem como na literatura cinza.

Todos os dados obtidos foram descritos e apresentados no Fluxograma PRISMA, Figura 1 (Liberati et al., 2009). 
Figura 1: Fluxograma PRISMA dos artigos incluídos na revisão sistemática.
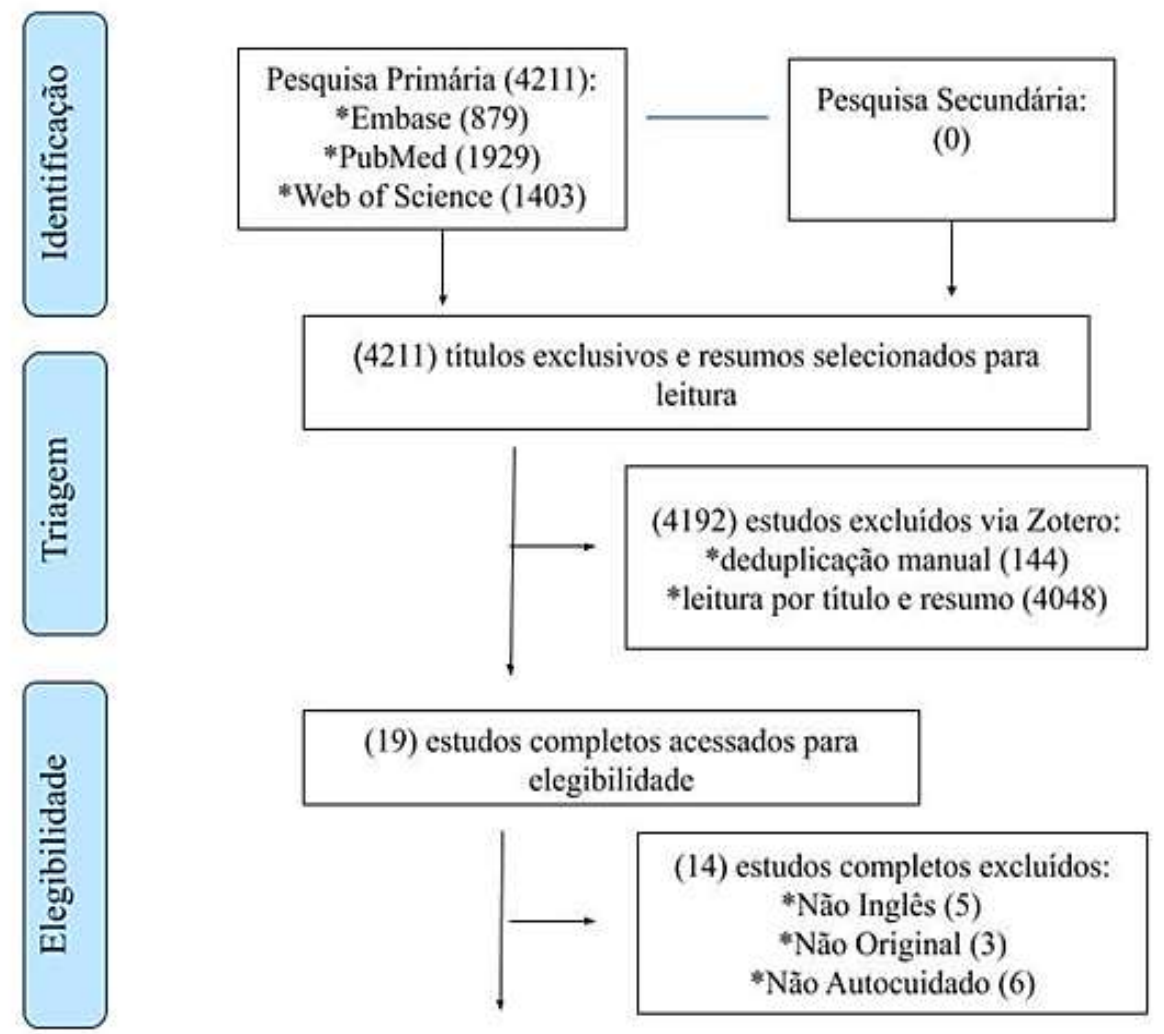

(19) estudos completos acessados para elegibilidade

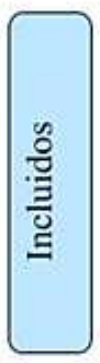

(5) estudos completos incluidos para elegibilidade

(14) estudos completos excluidos:

*Não Inglês (5)

"Não Original (3)

*Não Autocuidado (6)

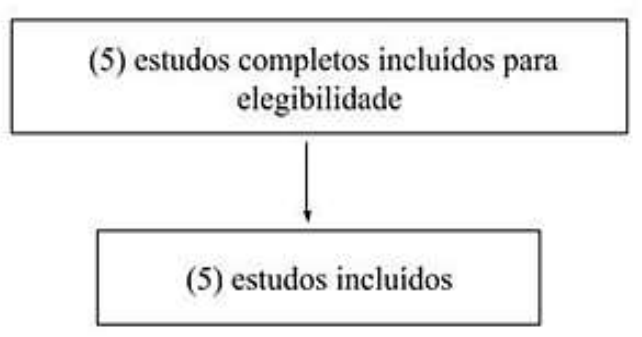

Fonte: Dados obtidos na pesquisa (2020)

\section{Estratégia para localizar os estudos}

Quatro revisores avaliaram de forma independente os títulos e resumos de todos os estudos originais identificados na busca eletrônica através da plataforma de referenciamento bibliográfico Zotero® (Zotero, 2020). Na plataforma, os estudos foram deduplicados e então separados em pastas para posterior leitura e análise pelo time de pesquisadores.

Os estudos que preencheram os critérios de inclusão desta RSL foram apresentados na Tabela 1. 
Tabela 1: Descrição dos estudos que atenderam aos critérios de inclusão.

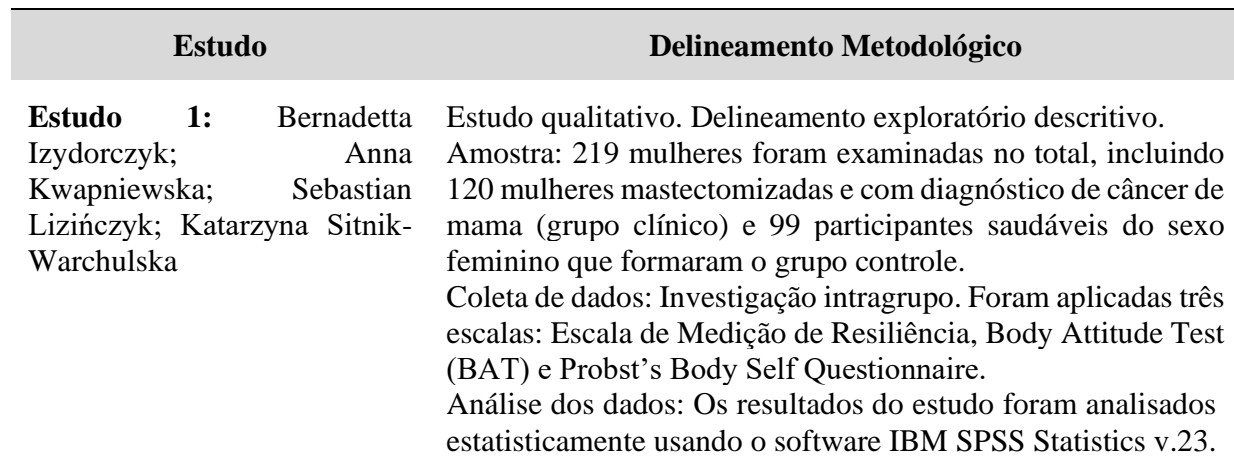

Estudo 2: Leticia Valente Dias Rosani Manfrin Muniz Aline da Costa Viegas Daniela Habekost Cardoso Debora Eduarda Duarte do Amaral Bruna Knob Pinto

Estudo 3: Mary Bredin

Estudo 4: Olalekan Olasehinde; Olujide Arije; Funmilola Olanike Wuraola et al

Estudo 5: NE Stecher, MA Cohen, EJ Myburgh
Estudo qualitativo. Delineamento exploratório descritivo.

Amostra: 6 mulheres que realizaram mastectomia devido ao câncer de mama.

Coleta de dados: semi-entrevista estruturada, contendo questões relacionadas às atividades diárias.

Análise dos dados: baseada nos preceitos da proposta operacional, com as seguintes etapas: ordenação dos dados, classificação dos dados, análise final e relatório final.

Estudo qualitativo. Delineamento exploratório descritivo. Amostra: o tamanho da amostra foi limitado a três participantes por causa do tempo necessário para realizar a intervenção de massagem.

Coleta de dados: entrevistas semiestruturadas de 1 hora e seis sessões de massagem terapêutica. A entrevista inicial e sessões de massagem subsequentes foram conduzidas pelo autor.

Análise de dados: foi realizada seguindo os métodos de pesquisa de Guba \& Lincoln.

Estudo qualitativo. Delineamento exploratório descritivo.

Amostra: Um total de 15 entrevistas foram realizadas porque este foi o ponto em que a saturação de dados foi atingida.

Coleta de dados: Um guia de entrevista foi desenvolvido a partir de uma revisão da literatura.

Análise dos dados: Todas as entrevistas foram transcritas na íntegra, e aquelas conduzidas no dialeto local foram traduzidos para o inglês para análise. A estrutura analítica e os códigos foram desenvolvidos usando Software ATLAS.ti 7.

Estudo qualitativo. Fenomenologia descritiva.

Amostra: grupo composto por 7 mulheres.

Coleta de dados: Com a orientação de um grupo local de apoio ao câncer de mama, entrevistas semiestruturadas foram conduzidas em inglês.

Análise de dados: As entrevistas transcritas foram analisadas tematicamente. Esta incluiu familiarização e imersão de dados, induzindo temas, codificação, elaboração e interpretação e verificação.

\section{Objetivo Geral}

Examinar as diferenças intragrupo relacionadas à imagem corporal e a resiliência psicológica das mulheres que realizaram mastectomia.

Examinar as experiências e vivências das mulheres que realizaram mastectomias devido ao câncer de mama durante a realização de atividades diárias.

Avaliar as experiências das mulheres que sofreram mastectomia, ressaltando as questões de imagem corporal das mesmas. As intervenções de massagem foram utilizadas no intuito de ajudá-las a se adaptarem melhor às mudanças de sua auto imagem.

Analisou, por meio das entrevistas, os principais temas relacionados aos impactos da mastectomia na vida das mulheres, tanto no âmbito físico quanto no psicológico, como as alterações na imagem corporal.

Examinar, por meio das entrevistas, o impacto psicológico do diagnóstico e do tratamento do câncer de mama, a alteração da imagem corporal e o apoio da família.

Fonte: Autores (2021).

\section{Restrições associadas ao estudo}

Não utilizamos restrições quanto ao ano de publicação para as buscas. A única restrição aplicada foi concernente à língua de publicação dos estudos.

\section{Idiomas das publicações aceitas para o estudo}

Utilizamos critério de língua inglesa para inclusão dos estudos da RSL. 


\section{Método das fontes de buscas}

O método usado para definir as fontes de buscas dos estudos foi construído através do uso de palavras-chaves previamente definidas segundo a pergunta de pesquisa "Como é a experiência de autocuidado de mulheres com câncer de mama após a mastectomia?” alinhada à estratégia PICOS, descrita no Quadro 2 (Santos et al., 2007).

Quadro 2: Estratégia PICOS.

\begin{tabular}{|lll|}
\hline Critérios investigados & Nomenclatura dos critérios & Descrição dos critérios \\
População & $\mathrm{P}=$ Population & mulheres com câncer de mama \\
Intervenção & $\mathrm{I}=$ Intervention & mastectomia \\
Comparação & $\mathrm{C}=$ Comparation & não se aplica \\
Resultado & $\mathrm{O}=$ Outcome & autocuidado \\
Tipo de Estudo & $\mathrm{S}=$ Study & estudo qualitativo \\
\hline
\end{tabular}

Fonte: Autores (2021).

Cabe ressaltar que a estratégia de busca foi adequada a cada plataforma, e para isso utilizamos termos indexados e seus respectivos sinônimos segundo o DeCs (Descritores em Ciências da Saúde, 2017), conforme descrito no item 9.

\section{Estratégia de buscas nas bases de dados elegíveis}

Para confeccionar as estratégias de buscas utilizamos um mapa conceitual (the concept map) para nortear as buscas, representado no Quadro 3.

\section{PUbMED:}

Estratégia de busca: (((((breast neoplasm) OR breast cancer) OR malignant neoplasm of breast) OR cancer of the breast)) AND $((((()(((($ self-management) OR self-management) OR self-care) OR management, self) OR care, self) OR help-seeking behavior) OR coping behavior)) OR mastectomy) OR surgical oncology) OR surgical procedures, operative)) AND (((c((()((qualitative research) OR qualitative study) OR qualitative analysis) OR thematic analysis) OR phenomenology) OR cultural anthropology) OR hermeneutics) OR focus group) OR grounded theory) OR ethnography).

\section{Web of Science:}

Estratégia de busca: (TS=(malignant neoplasm of breast) $\mathrm{OR} T \mathrm{TS}=($ cancer of the breast) $\mathrm{OR}$ TS=(breast neoplasms) OR $\mathrm{TS}=$ (breast cancer) $)$ AND $(\mathrm{TS}=$ (mastectomy) $\mathrm{OR} \mathrm{TS}=($ surgical oncology) OR TS=(surgical procedures, operative) $\mathrm{OR}$ TS=(self management) OR TS=(self-care) OR TS=(self-management) OR TS=(self-care) OR TS=(management, self) $\mathrm{OR} \mathrm{TS}=$ (care, self) OR TS=(help-seeking behavior) OR TS=(coping behavior)) AND (TS=(qualitative research) OR TS=(qualitative study) OR $\mathrm{TS}=$ (qualitative analysis) $\mathrm{OR} \mathrm{TS}=$ (thematic analysis) $\mathrm{OR} \mathrm{TS}=$ (phenomenology) $\mathrm{OR} \mathrm{TS}=($ cultural anthropology) $\mathrm{OR}$ $\mathrm{TS}=$ (hermeneutics) $\mathrm{OR} \mathrm{TS}=($ focus group) $\mathrm{OR} \mathrm{TS}=($ grounded theory $) \mathrm{OR} \mathrm{TS}=($ ethnography) $\mathrm{OR} \mathrm{TS}=($ feminism)$)$.

\section{Embase:}

Estratégia de busca: ('breast tumor'/exp OR 'breast cancer'/exp OR 'breast neoplasms pathology'/exp) AND ('mastectomy'/exp OR 'surgical oncology'/exp OR 'surgery'/exp OR 'self-care'/exp OR 'self-management support'/exp OR 'help seeking behavior'/exp OR 'coping behavior'/exp) AND ('qualitative analysis'/exp OR 'qualitative methods'/exp OR 'qualitative 
research'/exp OR 'thematic analysis'/exp OR 'phenomenology'/exp OR 'cultural anthropology'/exp OR 'hermeneutics'/exp OR 'focus group'/exp OR 'grounded theory'/exp OR 'ethnography'/exp OR 'feminism'/exp).

Quadro 3: The Concept Map.

\begin{tabular}{|cccc|}
\hline P (população) & $\begin{array}{c}\text { CONTEXTO =I (intervenção) }+ \\
\text { O (resultado) }\end{array}$ & S (estudo) \\
\hline $\begin{array}{c}\text { malignant neoplasm of } \\
\text { breast }\end{array}$ & mastectomy & self-management & qualitative research \\
\hline cancer of the breast & surgical oncology & self management & qualitative study \\
\hline breast neoplasms & surgical procedures, operative & management, self & qualitative analysis \\
\hline breast cancer & self-care & thematic analysis \\
\hline & care, self & phenomenology \\
\hline & self care & cultural anthropology \\
\hline & help-seeking behavior & hermeneutics \\
\hline & coping behavior & focus group \\
\hline & & grounded theory \\
\hline
\end{tabular}

Fonte: Autores (2021).

\section{Listagem das fontes de buscas}

A listagem das bases de dados utilizadas na RSL está apresentada na Quadro 4, abaixo.

Quadro 4: Bases de Dados Pesquisadas

\begin{tabular}{|cc|}
\hline Base de dados & Link de acesso \\
Pub Med & https://pubmed.ncbi.nlm.nih.gov/advanced/ \\
Embase & https://www.embase.com/a/\#/picoSearch \\
Web of Science & http://apps.webofknowledge.com/WOS_AdvancedSearch_input.do?SID=7Dq1vvWmRVsu6UGQ \\
& FWW\&product=WOS\&search_mode=AdvancedSearch \\
\hline
\end{tabular}

Fonte: Autores (2021).

\section{Tipos de artigos selecionados}

Foram selecionados os estudos originais com enfoque na metodologia qualitativa que abordaram o autocuidado de mulheres com câncer de mama após a mastectomia.

\section{Critérios de inclusão e exclusão dos artigos}

\section{Critérios de inclusão:}

a. Trabalhos publicados e disponíveis integralmente em bases de dados científicas de livre acesso à Universidade Federal de Uberlândia; 
b. Trabalhos originais;

c. Trabalhos em língua inglesa;

d. Trabalhos com publicação que já possuíam aprovação pela comunidade científica;

e. Trabalhos que abordavam o autocuidado de mulheres com câncer de mama após mastectomia como temática central e principal.

\section{Critérios de exclusão:}

Trabalhos não disponíveis integralmente nas bases de dados pesquisadas.

\section{Critérios de qualidade dos estudos primários}

Os critérios de qualidade dos estudos selecionados foram analisados e definidos através de consenso entre os pares de pesquisadores. Quando houve dúvida em relação à classificação do estudo em elegível ou inelegível procedeu-se ao desempate por consenso do time. Esse fluxo aconteceu de maneira informal pelo time e, portanto, não houve um registro formalizado para tal finalidade.

\section{Processo de seleção dos estudos primários}

Foram realizadas buscas com as palavras-chaves nas fontes de pesquisa definidas pelo time de pesquisadores. Dos trabalhos recuperados foram lidos à priori os resumos, e também já iniciamos uma pré-avaliação baseada nos critérios de inclusão e exclusão, com o intuito de selecionar os estudos que seriam lidos na íntegra posteriormente. Os estudos selecionados foram lidos integralmente e avaliados pelo time, neste momento a leitura foi cega e realizada por dois pesquisadores do time, onde foram averiguados, rigorosamente, de acordo com os critérios de inclusão e exclusão previamente definidos, sendo considerados elegíveis e inelegíveis para os objetivos da RSL.

\section{Estratégia de extração dos dados}

Dois revisores extraíram os dados dos estudos que responderam aos critérios de inclusão descritos anteriormente. Um formulário padronizado foi construído no Excel para extrair as informações concernentes às características metodológicas dos estudos, as características demográficas das participantes e os temas extraídos dos resultados dos estudos elegíveis.

\section{Procedimentos para análises}

Os estudos elegíveis foram analisados de forma conceitual utilizando como pano de fundo a análise temática (Braun \& Clarke, 2006). Os resultados obtidos constam na Tabela 2, abaixo. 
Tabela 2: Descrição dos temas apresentados em cada estudo elegível.

\begin{tabular}{lllll}
\hline \multicolumn{2}{c}{ Temas dos estudos incluídos } & Tema 3 & Tema 4 \\
\hline Estudo (ano) & Tema 1 & Tema 2 & Família & Efeitos do tratamento \\
\hline Dias et al., 2017 & Capacidade funcional & Autocuidado & Atividades cotidianas & Apoio familiar \\
\hline Bredin, 1999 & Imagem corporal & Impacto psicológico & Impacto na vida sexual & Capacidade funcional \\
\hline $\begin{array}{l}\text { Izydorczyk et al., } \\
\mathbf{2 0 1 9}\end{array}$ & $\begin{array}{l}\text { Resiliência } \\
\text { psicológica }\end{array}$ & Imagem corporal & Impacto psicológico & Autoavaliação \\
\hline $\begin{array}{l}\text { Olasehinde et al., } \\
\mathbf{2 0 1 9}\end{array}$ & Imagem corporal & Impacto na vida sexual & Impacto psicológico & Resiliência psicológica \\
\hline
\end{tabular}

Fonte: Autores (2021).

\section{Sumarização dos resultados}

Os resultados obtidos na RSL, estão apresentados na Tabela 3, onde descrevemos cada estudo elegível e seus principais resultados.

Tabela 3: Descrição dos principais resultados encontrados nos estudos elegíveis.

\begin{tabular}{|c|c|}
\hline Estudos elegíveis & Principais resultados encontrados \\
\hline Stecher et al. (2019) & $\begin{array}{l}\text {-A estrutura familiar beneficia o paciente e também os membros que se empenham a adaptar- } \\
\text { se entre si; } \\
\text {-Retrata que cada método terapêutico tem uma implicação, e em muitas situações a terapêutica } \\
\text { pode ser suspensa até que algum entrave clínico seja resolvido. }\end{array}$ \\
\hline Bredin (1999) & $\begin{array}{l}\text {-As mulheres demonstraram compreender a mama como um símbolo corpóreo da aparência } \\
\text { feminina, além de terem a necessidade de se considerar normal perante a sociedade; } \\
\text {-Processo familiar é necessário incluir também atividades de aconselhamento de casais, acerca } \\
\text { de questões sexuais; } \\
\text {-Autonomia é chave para a manutenção do autocuidado. }\end{array}$ \\
\hline Izydorczyk et al. (2019) & $\begin{array}{l}\text {-Enfrentamento após a mastectomia inicia-se pela dificuldade de lidar com as emoções; } \\
\text { Recuperação advinda da resiliência psicológica a partir do processo da autoavaliação utiliza o } \\
\text { autocuidado como estratégia de habilidade de enfrentamento particular. }\end{array}$ \\
\hline Olasehinde et al. (2019) & $\begin{array}{l}\text {-As mulheres demonstraram compreender a mama como um símbolo corpóreo da aparência } \\
\text { feminina, além de terem a necessidade de se considerar normal perante a sociedade; } \\
\text {-Processo familiar é necessário incluir também atividades de aconselhamento de casais, acerca } \\
\text { de questões sexuais; } \\
\text {-Recuperação advinda da resiliência psicológica a partir do processo da autoavaliação utiliza } \\
\text { o autocuidado como estratégia de habilidade de enfrentamento partícula. }\end{array}$ \\
\hline Dias et al. (2017) & $\begin{array}{l}\text {-Enfrentamento após a mastectomia inicia-se pela dificuldade de lidar com as emoções; } \\
\text {-A estrutura familiar beneficia o paciente e também os membros que se empenham a adaptar- } \\
\text { se entre si; } \\
\text {-Autonomia é chave para a manutenção do autocuidado; } \\
\text {-Ressalta a relevância do papel dos profissionais de saúde em colaborar com o bem-estar da } \\
\text { mulher mastectomizada. }\end{array}$ \\
\hline
\end{tabular}

Fonte: Autores (2021).

\section{Considerações Finais}

As principais etapas para o planejamento e execução de uma revisão sistemática de literatura foram relacionadas e explanadas neste artigo, com o intuito de demonstrar um "step by step" para a construção de revisões. Cabe frisar que existem outros tipos de revisão sistemática e, também, outras formas de executar essas revisões. 
Destarte, acreditamos que o método descrito neste artigo poderá corroborar os profissionais de saúde e acadêmicos a construírem de forma mais coesa e sistemática uma revisão de literatura, além de facilitar o trabalho em equipe durante o processo de confecção da mesma.

\section{Referências}

Antes, G., Herrle, F., Meerpohl, J. J., \& Von Elm, E. (2012). Scientific value of systematic reviews: survey of editors of core clinical journals. PLoS One. May;7(5):e35732.

Baek, S., Cho, J., Lim, K., Seo, Y., Yoon, D., \& Yun. (2018). The most downloaded and most cited articles in radiology journals: a comparative bibliometric analysis. European Radiology, v. 28, n. 11, p. 4832-4838.

Botelho, L. L. R., Cunha, C. C. A., \& Macedo, M. (2011). O método da revisão integrativa nos estudos organizacionais. Gest Soc. ago;5(11):121-36.

Braun, V., \& Clarke, V. (2006). Using thematic analysis in psychology. Qualitative Research in Psychology, 3 (2), 77-101. https://www.tandfonline.com/doi/abs/10.1191/1478088706qp063oa.

Bredin, M. (1999). Mastectomy, body image and therapeutic massage: A qualitative study of women's experience. Journal of advanced nursing, 295, 11131120

Centre for Reviews and Dissemination. (2009). Systematic reviews: CRD's guidance for undertaking reviews in health care [Internet]. York: CRD, University of York; 281 p. http://www.york.ac.uk/inst/crd/pdf/Systematic_Reviews.pdf

Chalmers, I., Enkin, M., \& Keirse, M. J. N. C. (1989). Effective care in pregnancy and childbirth. Oxford: Oxford University Press.

Cook, D. J., Mulrow, C. D., \& Haynes R. B. (1997). Revisões sistemáticas: síntese das melhores evidências para decisões clínicas. Ann Intern Med, 126 (5), 376-80. https://pubmed.ncbi.nlm.nih.gov/9054282/.

Descritores em Ciências da Saúde: DeCS. *. ed. rev. e ampl. (2017). BIREME / OPAS / OMS. http://decs.bvsalud.org.

Dias, L. V., Muniz, R. M., Viegas, A. D. C., Cardoso, D. H., Amaral, D. E. D. do, \& Pinto, B. K. (2017). Mastectomized woman in breast cancer: Experience of everyday activities. Mulher mastectomizada por câncer de mama: vivência das atividades cotidianas. Revista de Pesquisa: Cuidado é Fundamental Online, 94, 1074. Doi.org/10.9789/2175- 5361.2017.v9i4.1074-1080.

Galvão, M. C. B, \& Ricarte, I. L. M. (2020). Revisão sistemática da literatura: conceituação, produção e publicação. LOGEION: Rio de Janeiro, 6 (1), 57-73. https://doi.org/10.21728/logeion.2019v6n1.p57-73.

Galvão, M. C. B.; Ricarte, I. L. M., \& Pluye, P. (2017). Métodos de pesquisa mistos e revisões de literatura mistas: conceitos, construção e critérios de avaliação. InCID: Revista de Ciência da Informação e Documentação, 8(2), 4-24. https://www.revistas.usp.br/incid/article/view/121879.

Galvão, T. F., \& Pereira, M. G. (2014). Revisões sistemáticas da literatura: passos para sua elaboração. Epidemiologia e Serviços de Saúde, 23(1), 183-184. http://scielo.iec.gov.br/scielo.php?script=sci_arttext\&pid=S1679-49742014000100018\&lng=pt\&tlng=pt.

Higgins, J. P. T, \& Green, S. (2011). Cochrane Handbook for Systematic Reviews of Interventions Version 5.1.0. http://handbook.cochrane.org.

Izydorczyk, B., Kwapniewska, A., Lizińczyk, S., \& Sitnik- Warchulska, K. (2019). Characteristics of psychological resilience and body image in women in the early and late periods after mastectomy. Health Psychology Report, 71, 32-47. https://doi.org/10.5114/hpr.2018.79996

Liberati, A., Altman, D. G, Tetzlaff, J., Mulrow, C., Gotzsche, P.C, \& Ioannidis, J. P. A et al. (2009). The PRISMA statement for reporting systematic reviews and meta-analyses of studies that evaluate healthcare interventions: Explanation and elaboration. BMJ, 339. https://www.bmj.com/content/339/bmj.b2700.

Moloney, R., \& Maggs, C. (1999). Uma revisão sistemática das relações entre o planejamento manual de cuidados de enfermagem, manutenção de registros e resultados do paciente. J Adv Nurs, 30 (1), 51-7. https://pubmed.ncbi.nlm.nih.gov/10403980/.

Olasehinde, O., Arije, O., Wuraola, F. O., Samson, M., Olajide, O., Alabi, T., Arowolo, O., Boutin-Foster, C., Alatise, O. I., \& Kingham, T. P. (2019). Life Without a Breast: Exploring the Experiences of Young Nigerian Women After Mastectomy for Breast Cancer. Journal of Global Oncology, 5, 1-6. https://doi.org/10.1200/JGO.18.00248.

Santos, C. M. D. C., Pimenta, C. A. D. M., \& Nobre, M. R. C. (2007). A estratégia PICO para a construção da pergunta de pesquisa e busca de evidências. Revista Latino-Americana de Enfermagem, 15 (3), 508-511. https://www.redalyc.org/articulo.oa?id=2814/281421874023.

Stecher, N., Cohen, M., \& Myburgh, E. (2019). Experiences of women in survivorship following mastectomy in the Cape Metropole. South African Journal of Surgery, 19-23. https://doi.org/10.17159/2078-5151/2019/v57n1a2746.

The Cochrane Collaboration. (2012). History [Internet]. Oxford (UK): The Cochrane Collaboration; http://www.cochrane.org/about-us/history

The James Lind Library. (2007). Up-to-date, systematic reviews of all relevant, reliable evidence. http://www.jameslindlibrary.org/essays/interpretation/up-todate-systematic-reviews-of-all-relevant-reliable-evidence.html Pearson K. Report on certain enteric fever inoculation statistics. BMJ. 1904 Nov;2(2288):12436.

Zotero. (2020). Roy Rosenzweig Center for History and New Media. 